Journal of

Molecular Microbiology

and Biotechnology
J Mol Microbiol Biotechnol 2003;5:240-251

DOI: $10.1159 / 000071076$

\title{
Evaluation of Two-Dimensional Difference Gel Electrophoresis for Protein Profiling
}

\author{
Soluble Proteins of the Marine Bacterium Pirellula sp. Strain 1
}

\author{
Dörte Gade $^{a}$ Jürgen Thiermann ${ }^{b}$ Dieter Markowsky ${ }^{b}$ Ralf Rabus ${ }^{a}$ \\ a Max Planck Institute for Marine Microbiology, Bremen, and ${ }^{\mathrm{b}}$ Amersham Biosciences Europe GmbH, \\ Freiburg, Germany
}

\section{Key Words}

Two-dimensional gel electrophoresis - 2D DIGE •

DeCyder $^{\mathrm{TM}}$. SYPRO ${ }^{\circledR}$ Ruby · CyDyes ${ }^{\mathrm{TM}}$ - Silver staining .

Coomassie $\cdot$ Pirellula $\cdot$ Carbohydrates

\begin{abstract}
Two-dimensional gel electrophoresis (2DE) is a central tool of proteome research, since it allows separation of complex protein mixtures at highest resolution. Quantification of gene expression at the protein level requires sensitive visualization of protein spots over a wide linear range. Two-dimensional difference gel electrophoresis (2D DIGE) is a new fluorescent technique for protein labeling in 2DE gels. Proteins are labeled prior to electrophoresis with fluorescent CyDyes ${ }^{\mathrm{TM}}$ and differently labeled samples are then co-separated on the same 2DE gel. We evaluated 2D DIGE for detection and quantification of proteins specific for glucose or N-acetylglucosamine metabolism in the marine bacterium Pirellula sp. strain 1. The experiment was based on 10 parallel 2DE gels. Detection and comparison of the protein spots were performed with the DeCyder ${ }^{\mathrm{TM}}$ software that uses an internal standard to quantify differences in protein abundance with high statistical confidence; 24 proteins differing in abundance by a factor of at least 1.5 (t test value
\end{abstract}

$<10^{-9}$ ) were identified. For comparison, another experiment was carried out with four SYPRO ${ }^{\circledR}$-Ruby-stained 2DE gels for each of the two growth conditions; image analysis was done with the ImageMaster ${ }^{\mathrm{TM}}$ 2D Elite software. Sensitivity of the CyDye fluors was evaluated by comparing Cy2, Cy3, Cy5, SYPRO Ruby, silver, and colloidal Coomassie staining. Three replicate gels, each loaded with $50 \mu \mathrm{g}$ of protein, were run for each stain and the gels were analyzed with the ImageMaster software. Labeling with CyDyes allowed detection of almost as many protein spots as staining with silver or SYPRO Ruby.

Copyright @ 2003 S. Karger AG, Basel

\section{Introduction}

The advent of genomics revolutionized the possibilities of biological experimentation. With the entire genetic information of a given study organism available, analysis of gene expression is not limited to selected genes anymore, but can be performed on the global level. This opens new avenues for functional analysis of the complexity of physiological and cellular aspects of living cells. Cells regulate gene expression, in order to adapt to changing environmental conditions in an economical manner.

\section{KARGER}

Fax +41613061234

E-Mail karger@karger.ch

www.karger.com

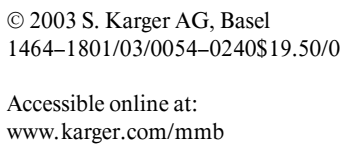

Dr. Ralf Rabus

MPI for Marine Microbiology

Celsiusstrasse 1

DE-28359 Bremen (Germany)

Tel. +49 4212028 736, Fax +49 4212028 538, E-Mail rrabus@mpi-bremen.de 


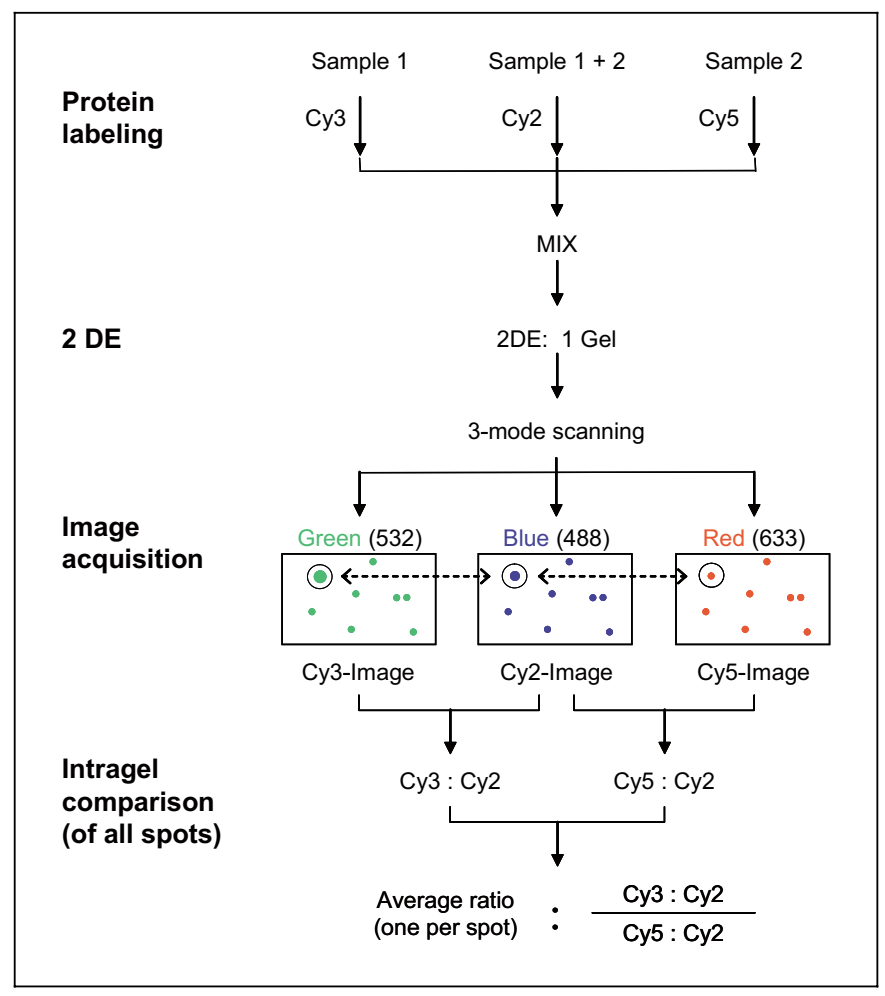

Fig. 1. Schematic representation of the $2 D$ DIGE workflow for the differential analyses of protein abundance in two different samples (1 and 2). (1) The two samples are labeled with $\mathrm{Cy} 3$ and $\mathrm{Cy} 5$, respectively. Equal amounts of both samples are mixed and labeled with Cy2 to be used as internal standard. (2) The three labeling preparations are then mixed prior to simultaneous electrophoretic separation on the same 2DE gel. (3) For each of the three cyanine fluorophores an independent image is acquired by successive scanning of the gel three times with the fluorophore-specific excitation and emission wavelengths (table 2). (4) During intragel comparison, for each spot the ratios of normalized volumes between samples and internal standard are determined. Differences in protein abundance are then determined by calculating the average ratios [(Cy3:Cy2):(Cy5:Cy2)]. Statistical confidence is achieved by analysis of parallel gels (intergel comparison).

mixed and labeled with Cy2. The labeled samples and the internal standard are mixed prior to electrophoresis. (2) $2 \mathrm{DE}$ is essentially carried out according to conventional protocols. Since the CyDyes carry a positive charge that compensates for the positive charge of the lysine amino group lost during formation of the amide bond (termed charge matching), only little shift of the $p I s$ is observed relative to the unlabeled proteins. Labeling with any of the three CyDyes will increase the molecular mass of the proteins by approximately $500 \mathrm{Da}$. This shift is most pronounced with proteins of low molecular weight. Since these shifts in molecular mass are almost identical for the three CyDyes, they do not affect image analysis. (3) Image acquisition is based on the different excitation and emission wavelengths of the three cyanine fluorophores (table 2). Thus, from the different samples run on the same gel, separate images are generated, which are devoid of gel-to-gel variations. (4) Therefore, the different images of one gel can be compared without warping. Amersham Biosciences developed a specific software, DeCyder, for the analysis of gels generated by 2D DIGE. For each sample (labeled with $\mathrm{Cy} 3$ or $\mathrm{Cy} 5$ ), spot boundaries are defined separately. Both sets of spot boundaries (termed mapsets) are then superimposed on the Cy2-labeled internal standard. Thus using the two sample-specific mapsets, the ratios of spot volumes between an individual sample and the internal standard can be determined. Subsequently, for each spot, the change in protein abundance between the different samples can be calculated as the ratio of the two 'sample:internal standard' ratios [(C3:Cy2): (Cy5:Cy2)]. Using the 'Batch' modus of DeCyder software, parallel gels can be analyzed, allowing to determine changes in protein quantities with high statistical confidence. The 2D DIGE process allows to limit gel-to-gel variations and to reduce the number of gels.

First applications of the 2D DIGE method for biomedical systems [8-10] and the standard bacterium Escherichia coli $[11,12]$ have been reported only very recently. Here we apply, for the first time, the 2D DIGE system under optimal technological conditions for an environmental bacterium; i.e. all three available CyDyes are used, with Cy2 as label for the internal standard, the Typhoon ${ }^{\mathrm{TM}}$ 9400 scanner equipped with three lasers is used for image acquisition and the DeCyder software is used for differential in-gel analysis and batch processing. In this study we evaluated the 2D DIGE system for protein profiling in the marine bacterium Pirellula sp. strain 1, currently described as 'Rhodopirellula baltica'. This bacterium was isolated from the water column of the Kiel Fjord, Germany [13] and found to be catabolically specialized in the utilization of carbohydrates [Schlesner, Gade, Rabus, unpubl.]. Using a proteomic approach, we have recently identified a putative dehydrogenase which was specifically synthesized during growth with $\mathrm{N}$-acetylglucosamine [14]. The degradation of $\mathrm{N}$-acetylglucosamine is of environmental relevance in the marine system, since it is the monomer of one of the most abundant biopolymers, chitin [15]. Pirellula sp. strain 1 is assumed to be a representative of those bacteria that contribute significantly to the degradation of carbohydrates in marine systems. This bacterium has an unusually large genome size of approximately $7 \mathrm{Mb}$, the sequence of which has recently been 
determined by the REGX consortium (www.regx.de). Here we analyzed protein patterns of cells grown with $\mathrm{N}$ acetylglucosamine or glucose with the 2D DIGE system and with SYPRO Ruby staining. In addition, we compared the sensitivity of CyDyes with that of SYPRO Ruby, silver and cCBB, again using samples from $\mathrm{N}$-acetylglucosamine-grown cells.

\section{Materials and Methods}

\section{Media and Cultivation}

Pirellula sp. strain 1 was grown on mineral medium with $\mathrm{N}$ acetylglucosamine $(10 \mathrm{~m} M)$ or glucose $(10 \mathrm{mM})$ and ammonium $(1 \mathrm{~m} M)$ as described before [14]. The study organism is currently described as the type strain of a new genus and species, 'Rhodopirellula baltica' [Schlesner et al., unpubl.].

\section{Sample Preparation}

Cells from exponential cultures of Pirellula sp. strain 1 were harvested by centrifugation $\left(10,000 \mathrm{~g}, 20 \mathrm{~min}, 4^{\circ} \mathrm{C}\right)$. The pellets were washed with $100 \mathrm{~m} M$ Tris- $\mathrm{HCl} \mathrm{pH} 7.5$ containing $5 \mathrm{mM} \mathrm{MgCl} 2$. Cell pellets (about $100 \mathrm{mg}$ wet weight each) were immediately frozen in liquid nitrogen and stored at $-80^{\circ} \mathrm{C}$ until cell breakage and $2 \mathrm{DE}$. The pellets were resuspended in $1 \mathrm{ml}$ lysis buffer ( $7 M$ urea, $2 M$ thiourea, 2\% DTT, 2\% CHAPS, 0.5\% carrier ampholytes (Amersham Biosciences, Freiburg, Germany). Cell breakage was carried out by applying the PlusOne ${ }^{\circledR}$ Sample Grinding Kit from Amersham Biosciences following the instructions of the manufacturer. Cell debris, DNA and membranes were removed by centrifugation $\left(100,000 \mathrm{~g}, 1 \mathrm{~h}, 4^{\circ} \mathrm{C}\right)$ and the protein content of this fraction was determined by the method described by Bradford [16].

\section{Protein Labeling with Cyanine Dyes}

Pellets of Pirellula sp. strain 1 cells grown with glucose or Nacetylglucosamine were solubilized in lysis buffer (7 M urea, $2 M$ thiourea, $30 \mathrm{~m} M$ Tris-HCl, $2 \%$ CHAPS; final $\mathrm{pH} 8.5$ ). DTT and carrier ampholytes were omitted from the sample at this stage since the respective thiol and primary amine groups could potentially react with the NHS esters of the cyanine dyes. Cell breakage was carried out as described above. The $\mathrm{pH}$ of the samples was adjusted to 8.5 by careful addition of $50 \mathrm{mM} \mathrm{NaOH}$, as this has been shown to be the optimal $\mathrm{pH}$ for the labeling reaction [17]. The labeling was performed following the manufacturer's recommended protocol. Briefly, a stock solution of $1 \mathrm{ng}$ fluor dye/ $\mu$ l (in dimethyl formamide; DMF) was further diluted to a working solution of $400 \mathrm{pmol} / \mu \mathrm{l}$ in anhydrous DMF. Finally, $50 \mu \mathrm{g}$ of protein of either sample were labeled with $400 \mathrm{pmol}$ dye. Samples were vortexed and incubated on ice for $30 \mathrm{~min}$ in the dark. The reaction was stopped by adding $1 \mu 1$ of $10 \mathrm{~m} M$ lysine per $400 \mathrm{pmol}$ dye used. The samples were vortexed and incubated for $10 \mathrm{~min}$ on ice in the dark. An equal volume of $2 \times 2 \mathrm{DE}$ sample buffer (as lysis buffer above, plus $2 \%$ carrier ampholytes and $2 \%$ DTT) was added to the labeled protein yielding a final concentration of $7 M$ urea, $2 M$ thiourea, $2 \%$ CHAPS, $1 \%$ carrier ampholytes and $1 \%$ DTT. Labeled samples were combined and rehydration buffer (7 $M$ urea, $2 M$ thiourea, 2\% CHAPS, $0.4 \%$ DTT and $0.5 \%$ carrier ampholytes) was added to a final volume of $450 \mu$ l. This mixture was immediately used for isoelectric focussing. A pool of the two samples
Table 2. Emission filters and laser combinations used for detection of fluorescent labels

\begin{tabular}{lll}
\hline Fluorophore & Emission filter, nm & Laser \\
\hline Cy2 & 520 BPa 40 & Blue2 (488) \\
Cy3 & 580 BP 30 & Green (532) \\
Cy5 & 670 BP 30 & Red (633) \\
SYPRO Ruby & 610 BP 30 & Green (532) or Blue1 (457) \\
\hline
\end{tabular}

a $\quad \mathrm{BP}=$ Bandpass.

was prepared and labeled with Cy2 to be used as internal standard on each gel. For this, $25 \mu \mathrm{g}$ protein of each sample (cells grown with $\mathrm{N}$-acetylglucosamine and glucose, respectively) were mixed and the labeling was carried out as described above. For 2DE equal amounts of each of the three labeled preparations ( $\mathrm{N}$-acetylglucosamine, glucose, and internal standard composed of both) were mixed to be run on the same gel. The total amount of protein loaded on each gel was $150 \mu \mathrm{g}$.

\section{DE and Protein Visualization}

Isoelectric focussing was carried out as described before [18] using the IPGphor ${ }^{\mathrm{TM}}$ system and commercial 24-cm-long IPG strips (linear $\mathrm{pH}$ gradient of 4-7; Amersham Biosciences). Prior to SDSPAGE, IPG strips were equilibrated with DTT- and iodoacetamidecontaining buffers [2]. For second-dimension separation, the Ettan ${ }^{\mathrm{TM}}$ Dalt II system (Amersham Biosciences) was used. Gels were made of $375 \mathrm{~m} M$ Tris- $\mathrm{HCl}, 0.1 \%$ SDS and $12.5 \%$ Duracryl. Twelve parallel gels were run at $25^{\circ} \mathrm{C}$ with a running buffer composed of $25 \mathrm{mM}$ Tris, $192 \mathrm{~m} M$ glycine and $0.1 \%$ SDS. Electrophoresis was conducted at $180 \mathrm{~W}$ and stopped when the bromophenol blue dye front reached the bottom of the gel.

After SDS-PAGE, cyanine dye-labeled protein gels were scanned directly between the low-fluorescence glass plates using the Typhoon 9400 scanner (Amersham Biosciences). All gels were scanned with a resolution of $100 \mu \mathrm{m}$ and the photomultiplier tube set at $500 \mathrm{~V}$. Emission filters and laser combinations are listed in table 2.

Protein spots in gels with unlabeled samples were visualized using cCBB (modified from Doherty et al. [19]), Fast Blue Coomassie stain (Genomic Solutions, Ann Arbor, Mich., USA), silver (modified from Heukeshoven and Dernick [20]) and SYPRO Ruby (Molecular Probes Inc., Eugene, Oreg., USA). For comparison of protein detection with SYPRO Ruby and silver staining, SYPRO Ruby stained gels were destained over night in $10 \%(\mathrm{v} / \mathrm{v})$ methanol and $7 \%(\mathrm{v} / \mathrm{v})$ glacial acetic acid, and then stained with silver nitrate. Whereas images of SYPRO-Ruby-stained gels were also acquired with the fluorescence scanner Typhoon, those of silver- and cCBB-stained gels were collected with the Image Scanner (Amersham Biosciences).

\section{Image Analysis}

Determination of protein abundance and statistics based on 2D DIGE (table 3) were carried out with the DeCyder software package (version 4.0; Amersham Biosciences) according to the following steps. (1) From a single gel, three data sets are produced during scanning, corresponding to the $\mathrm{Cy} 2, \mathrm{Cy} 3$ and $\mathrm{Cy} 5$ images. First step of spot detection is the creation of electronically merged images for each 
of the $\mathrm{Cy} 3 / \mathrm{Cy} 2$ and $\mathrm{Cy} 5 / \mathrm{Cy} 2$ pairs. Spot maps are then created for each merged image (co-detection algorithm). Here the number of estimated spots can be defined by the user. Then the spotmap is superimposed onto the original image pair of a merged image. This results in identical boundaries for the same spots in the original images. (2) Normalized ratios of volumes (Cy3:Cy2 and Cy5:Cy2) are calculated for each spot and correlated to the gel-specific internal standard (Cy2). (3) For inter-gel comparison, corresponding spots were matched between all $20 \mathrm{Cy} 2$-images (10 each for Cy3:Cy2 and Cy5:Cy2 pairs, respectively). (4) After correlation with the internal standard across all Cy2 images, the average ratio of abundance was calculated for each spot. (5) Statistical analysis was performed by using the 'standardized log abundance'. The calculated t test value implicated the degree of statistical confidence of the observed differences in abundance. Intra-gel comparison would be facilitated if spot boundaries were defined for the Cy2-labeled internal standard and then superimposed on the individual samples labeled with either $\mathrm{Cy} 3$ or Cy5 (resulting in one instead of two mapsets).

For the analysis of the other gels the ImageMaster software (version 4.01; Amersham Biosciences) was used. When SYPRO-Rubystained gels were analyzed for comparison with the results obtained by 2D DIGE/DeCyder (see table 3), the following steps were applied. (1) The contrast of images was adjusted to allow optimal perception of spots. (2) Parameters of the 'spot detection wizard' were defined such that detection of artifacts (e.g. dust particles) was limited. The same parameters were applied for all eight gels analyzed. (3) Spot finding was manually edited to remove artifacts and to optimize spot boundaries (steps 1-3 were also carried out, when the sensitivity of the used stains was determined; fig. 4). (4) In the reference gel, the detection of spots was finally limited to those spots that were identified before by application of DeCyder software to differ in abundance, whereas in all other seven gels, the detection of all spots was conserved. (5) Then all gels were matched to the selected spots in the reference gel and the matches were manually edited. For example, if a spot could only be detected in the gel set representing one of the two studied growth conditions, its boundary was manually inserted in images of the other gel set where the spot was missing. This made it possible to calculate the spot volume ratios for all spots. (6) Background subtraction was carried out according to 'lowest on boundary'.

Gel-to-gel variations may cause differences in volumes of the same spots in parallel gels, despite the application of the same amount of total protein to each gel. These differences are leveled out by 'normalization', since they do not reflect biological changes. In the DeCyder software, normalization is performed after spot detection and calculation of ratios in abundance for each spot in all gels (for details, see ref. [12]). In case of 10 analyzed gels, a total of 20 Cy3:Cy2 and Cy5:Cy2 ratios was calculated for each spot. During standardization, all of these spot-specific ratios were related to one common value for the internal standard (Cy2), which is set at 0 on the $\log$ scale for abundance (fig. 3 , bottom horizontal panel). When ImageMaster software was used for image analyses, normalization was carried out differently. For each gel of a matchset, the sum of the total spot volumes was determined and set at $100 \%$. Then the shares of individual spot volumes in the sum of the total spot volumes of a gel were calculated. Share values for individual spots should be constant in a matchset, i.e. they should not be affected by varying staining intensities in parallel gels. Changes in protein abundance are then determined by calculating the ratio between sample-specific average share values.
Table 3. Ratios of protein abundance and statistical confidence of proteins identified as being differently abundant in cells grown with $\mathrm{N}$-acetylglucosamine versus glucose

\begin{tabular}{|c|c|c|c|}
\hline \multirow{2}{*}{$\begin{array}{l}\text { Spot } \\
\text { No. }^{\text {a }}\end{array}$} & \multicolumn{2}{|l|}{ 2D DIGE } & \multirow{2}{*}{$\begin{array}{l}\text { SYPRO Ruby } \\
\text { average ratio }^{d}\end{array}$} \\
\hline & average ratio ${ }^{b}$ & $\mathrm{t}$ test $^{\mathrm{c}}$ & \\
\hline 1 & 43.9 & $4.3 \cdot 10^{-19}$ & 12.0 \\
\hline 2 & 32.4 & $2.3 \cdot 10^{-14}$ & 36.5 \\
\hline 3 & 15.7 & $8.6 \cdot 10^{-13}$ & 5.9 \\
\hline 4 & 4.5 & $1.1 \cdot 10^{-20}$ & 3.8 \\
\hline 5 & 2.9 & $1.5 \cdot 10^{-12}$ & 1.9 \\
\hline 6 & 2.2 & $3.6 \cdot 10^{-13}$ & 1.6 \\
\hline 7 & 2.1 & $2.4 \cdot 10^{-12}$ & 2.0 \\
\hline 8 & 2.0 & $5.0 \cdot 10^{-13}$ & 1.8 \\
\hline 9 & 1.9 & $9.7 \cdot 10^{-18}$ & 1.5 \\
\hline 10 & 1.9 & $6.4 \cdot 10^{-10}$ & 1.0 \\
\hline 11 & 1.9 & $3.3 \cdot 10^{-14}$ & 2.6 \\
\hline 12 & 1.9 & $6.3 \cdot 10^{-12}$ & 2.7 \\
\hline 13 & 1.8 & $2.0 \cdot 10^{-12}$ & 1.5 \\
\hline 14 & 1.8 & $1.9 \cdot 10^{-14}$ & 2.1 \\
\hline 15 & 1.7 & $5.3 \cdot 10^{-14}$ & 1.6 \\
\hline 16 & 1.6 & $3.5 \cdot 10^{-10}$ & 1.6 \\
\hline 17 & 1.6 & $5.3 \cdot 10^{-10}$ & 1.8 \\
\hline 18 & -1.7 & $7.6 \cdot 10^{-12}$ & 1.2 \\
\hline 19 & -1.8 & $5.2 \cdot 10^{-11}$ & -2.1 \\
\hline 20 & -1.8 & $1.0 \cdot 10^{-13}$ & 1.1 \\
\hline 21 & -1.9 & $1.3 \cdot 10^{-13}$ & -1.4 \\
\hline 22 & -2.0 & $2.8 \cdot 10^{-14}$ & -2.7 \\
\hline 23 & -2.1 & $1.4 \cdot 10^{-14}$ & -2.0 \\
\hline 24 & -2.6 & $3.7 \cdot 10^{-14}$ & -2.8 \\
\hline
\end{tabular}

a Spot numbers are the same as in figure 2.

b Average ratios were calculated from 10 parallel 2D DIGE gels by the DeCyder software. Differential in-gel analysis was based on an 'estimated number of spots' of 2,500 as guide for spot detection. If for a batch of 5 gels, this number was increased to 4,000 , the values of the average ratio showed differences of $\pm 10 \%$ in some cases.

c Student's t test was used according to the null hypothesis. The probability is calculated that the abundance of a given protein spot under different conditions is the same.

d Average ratios were calculated from two set of gels (each containing 4 gels) by comparison of electronic average gels using the ImageMaster software.

Fig. 2. Cyanine fluorophore-specific images of a $2 \mathrm{DE}$ gel (2D DIGE) of protein samples of Pirellula sp. strain 1. Soluble proteins of cells grown with glucose $(\mathbf{A})$ and $\mathrm{N}$-acetylglucosamine (B), were labeled with $\mathrm{Cy} 5$ (A) and Cy3 (B), respectively. In both cases, $50 \mu \mathrm{g}$ of protein were used and isoelectric focussing was carried out with a $\mathrm{pH}$ gradient of 4-7. Both samples were simultaneously separated on the same gel. Excitation of the cyanine fluorophores and collection of the emitted fluorescent signals were as indicated in table 2 . Numbers indicate proteins that are differently abundant by a factor of at least 1.5 -fold ( $\mathrm{t}$ test value $<10^{-9}$ in 10 parallel gels) in the two studied growth conditions; numbers correspond to those used in figure 3 and table 3 (listing average ratios and statistical confidence). 

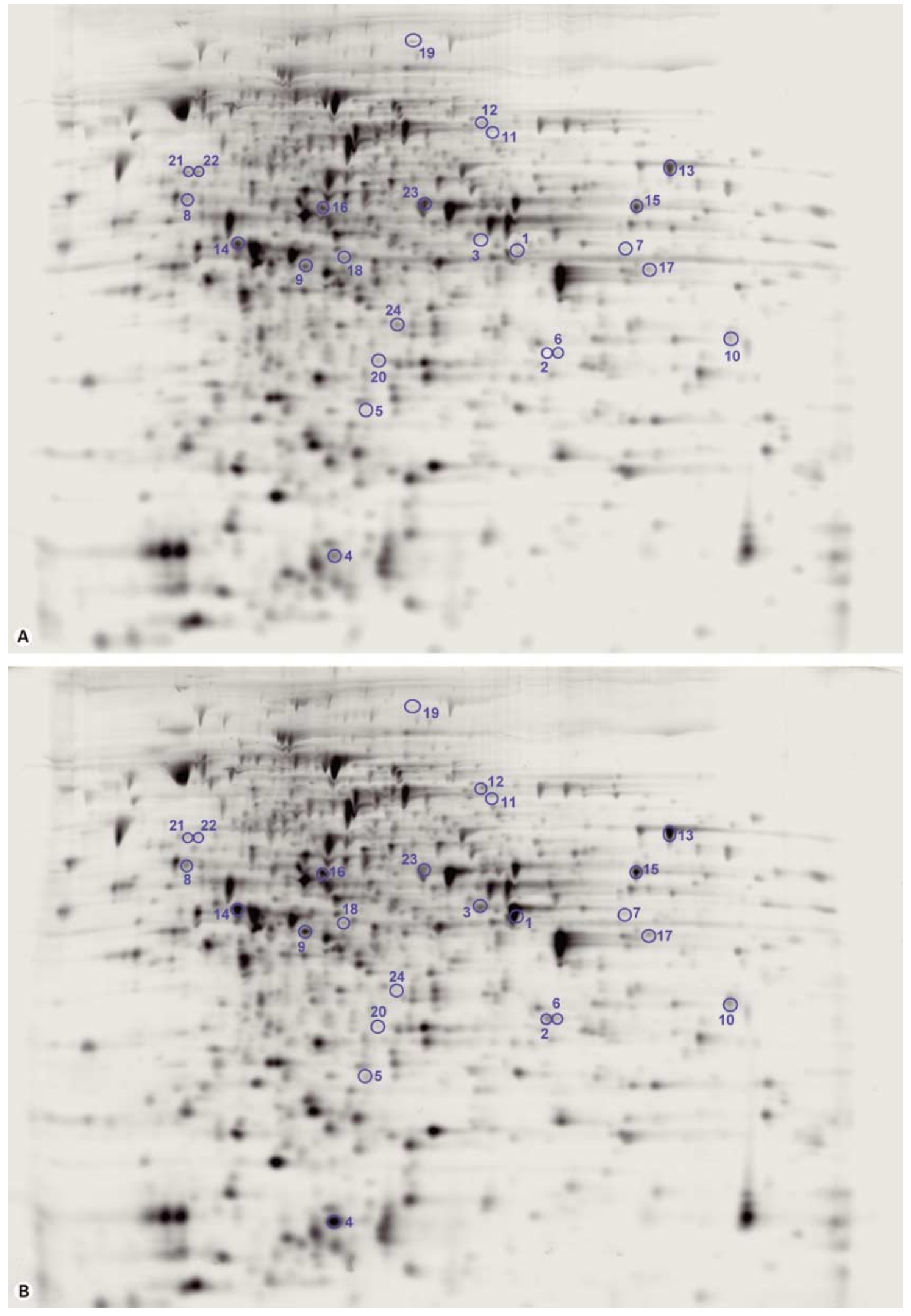


\section{Results and Discussion}

In a previous study we observed by $2 \mathrm{DE}$ analysis that cells of Pirellula sp. strain 1 grown with N-acetylglucosamine contain proteins that are apparently less abundant in cells grown with glucose [14]. In the present study we used this inducible $\mathrm{N}$-acetylglucosamine metabolism as a model system to evaluate the 2D DIGE system for detailed analysis of changes in protein quantities in this bacterium. Such a proteomic tool will be of great benefit for future studies with this bacterium, when global investigations are able to benefit from the fully sequenced genome.

\section{Determination of Changes in Protein Quantities Using} the 2D DIGE System

In this part of the study we analyzed a batch of 10 parallel gels, consisting of two subsets of 5 gels each. All of these gels were run simultaneously. In the first subset extracts of soluble proteins from $\mathrm{N}$-acetylglucosamine grown cells were labeled with $\mathrm{Cy} 3$, whereas protein extracts from glucose-grown cells were labeled with Cy5. This labeling order was reversed in the second subset of gels. In both subsets, a mixture of protein extracts from both types of samples was labeled with $\mathrm{Cy} 2$ and used as internal standard. By changing the labeling order for $\mathrm{Cy} 3$ and $\mathrm{Cy} 5$, we tried to take into account a possible bias resulting from the different CyDyes. The statistical analysis was carried out with 1,688 spots detected essentially in all gels using the DeCyder software. On the basis of a matchset from the 10 gels, changes in protein abundance were regarded as significant, when minimal values for the average ratio of 1.5 and maximal values for the t test of $10^{-9}$ were reached. With this conservative setting of parameters we determined 24 protein spots, the abundance of which differed in cells grown with $\mathrm{N}$-acetylglucosamine or glucose. Average ratios ranged from 1.5 to 44 and $t$ test values from $10^{-9}$ to $10^{-20}$ (fig. 2 , table 3 ). For all 24 proteins, consistency of matching was manually verified throughout the entire matchset. Details of the DeCyder output are exemplified for three spots (No. 1, 17, 23 ) in figure 3. For comparison, image analysis was carried out with reduced stringency for the statistical parameters. If the thresholds for average ratio and t test were altered to values of 1.2 and 0.05 , respectively, as previously reported in other studies [8, 17], approximately 387 spots appeared to have different abundance in the two analyzed growth conditions. Thus parameters for statistical stringency defined by the user have a major influence on the outcome of the image analysis. Despite the as- sumed analytical reliability of the large number of 387 differential protein spots (corresponding to approximately $23 \%$ of all detected proteins), a biological meaning should not be directly inferred. It should be taken into account that the only biological difference between the two analyzed samples is the substrate used for growth. From a physiological point of view, mainly alterations on the level of catabolic/anabolic enzymes and some regulatory proteins should occur. However, one would not expect this to translate into such high numbers of differentially abundant proteins. Assessment of the biological significance of the observed changes in protein quantities requires the identification of the respective proteins and further studies.

To assess a possible Cy3/Cy5-specific labeling bias we also analyzed both subsets of 5 gels each. Overall, the same protein spots were found to differ between the two growth conditions in the two subsets. Variations of the 24 protein spots selected before from the batch of 10 gels ranged between unchanged (1.0) and 1.4-fold ( 20 spots by 1.0 - to 1.2 -fold, 3 spots by 1.3 -fold and 1 spot by 1.4 -fold). Variations did not follow a consistent trend. Statistical analysis by means of analysis of variance (ANOVA; implemented in DeCyder) indicated that these variations were of no significance. It should be noted that ANOVA identified among the total of 1,688 detected protein spots only 3 , the abundance of which appeared to be influenced by the type of CyDye used for labeling. Thus no significant bias was introduced by the type of CyDye selected for labeling of a particular sample. Similar observations were recently reported for mouse liver cells [17]. In addition, we evaluated the influence of the number of analyzed gels

Fig. 3. Information on differently abundant proteins extracted from acquired gel images by DeCyder software. The three vertical panels (A-C) compile the information on the abundance of three selected spots $(1,17$ and 23$)$ in cells grown with glucose and $\mathrm{N}$-acetylglucosamine, respectively (spot numbering corresponds to that used in fig. 2 and table 3). The top horizontal panel displays partial views of the images of the 2DE gels shown in figure 2. Spot boundaries defined by DeCyder software are indicated as green lines. The spot boundaries of the selected protein spots are highlighted with purple lines. The middle horizontal panel displays the 3D images of the selected spots. The 3D images were rotated to allow optimal perception. The bottom horizontal panel shows scattered blot representations ('Graph View') of the log abundances of the selected spots under the two biological conditions (Glc, growth with glucose; NAG, growth with $\mathrm{N}$-acetylglucosamine) in the analyzed set of 10 parallel 2DE gels. The log of abundance is displayed at the y-axis and related to the normalized standard. 


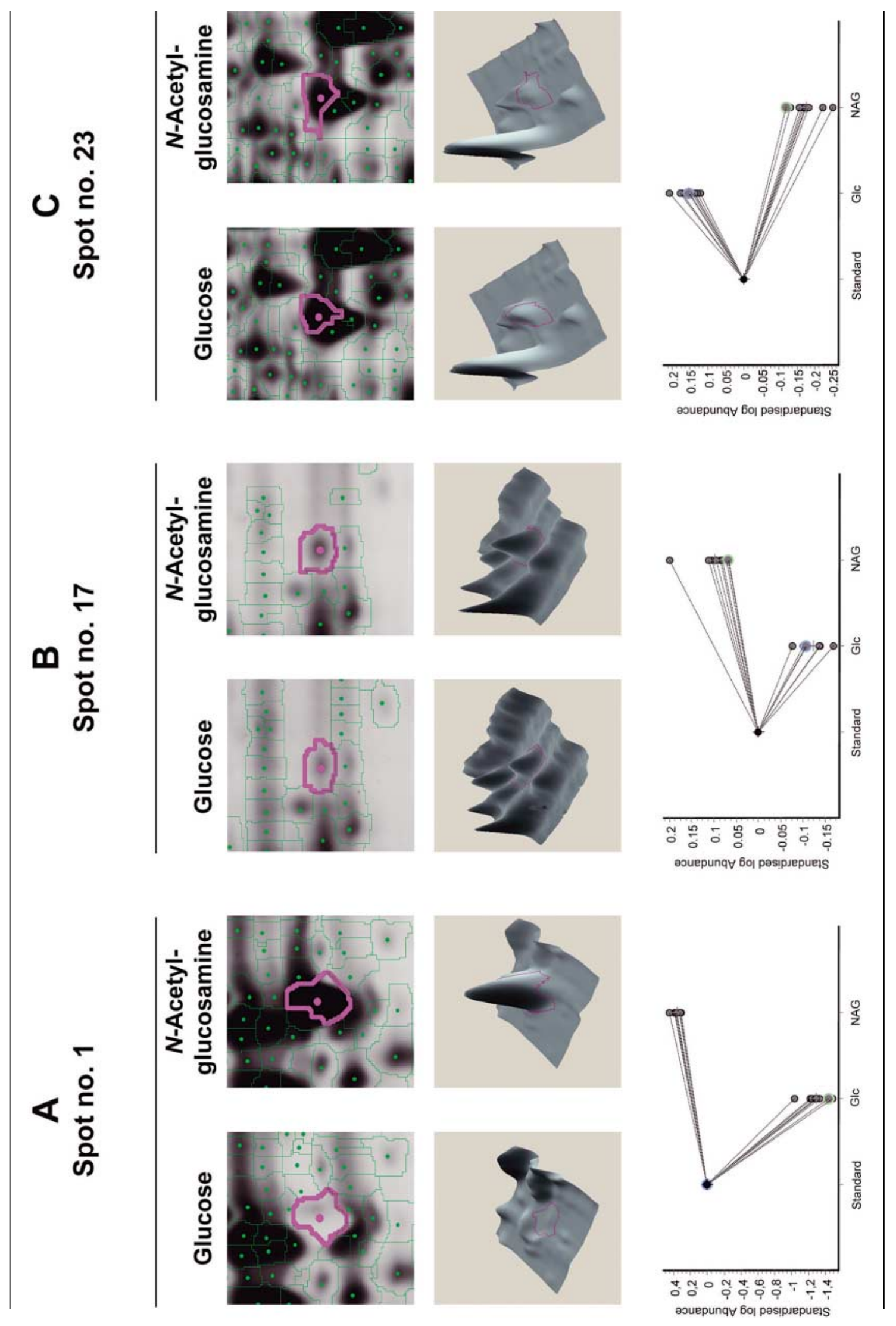

Evaluation of Two-Dimensional Difference

J Mol Microbiol Biotechnol 2003;5:240-251 
on statistical confidence. As expected, the $t$ test values increased if the number of parallel gels analyzed was reduced from 10 to 5 . The lowest $t$ test value observed for the 24 selected protein spots with either of the two subsets of gels was $10^{-5}$. This is significantly better than the 5 or $1 \%$ confidence levels usually applied in statistical analysis on the basis of the t test [21].

The statistical confidence of the differences in protein abundance described above resulted from the application of an internal standard. To analyze two different samples in addition to the internal standard in a single gel, the image acquisition has to be carried out successively at the three CyDye-specific wavelengths (table 2). If a scanner with three lasers is not available, but one with two, an internal standard can nevertheless be applied as described recently by Yan et al. [11]. In this study one sample was labeled with $\mathrm{Cy} 3$ and run together with a mixture of both samples labeled with Cy5 on a single gel. This procedure resulted in the high confidence achievable by the 2D DIGE system and image analysis with DeCyder. However, the use of a third CyDye (Cy2) allows reduction of the number of gels that have to be run.

Basic data generated and used by DeCyder software to calculate average ratio and t test values for changes in protein abundance can be visually inspected for each spot in each analyzed gel (fig. 3). Spot boundaries defined in the $\mathrm{Cy} 3$ and $\mathrm{Cy} 5$ channel can be displayed via zoom-in views of the corresponding gel areas to control matching. A 3D view allows to examine the $3 \mathrm{D}$ shape of spots and to evaluate the quality of electrophoretic separation and spot detection. A 'Graph View' displays the abundance of the selected protein spot under the analyzed conditions in all gels relative to the internal standard, providing a visual impression of the statistical confidence. The $3 \mathrm{D}$ view is especially useful for a refined control of spot boundaries in areas of insufficient electrophoretic separation (fig. 3B, C). In figure $3 \mathrm{C}$, the respective data are presented for spot No. 23. The 2D view of the corresponding gel section shows that spot No. 23 is part of a large spot. Only the 3D view allows recognition of spot No. 23 as a distinct spot incompletely separated from the neighboring spot. Such information is not directly provided by the ImageMaster software, but has to be manually retrieved by application of the 'Profile' tool displaying 2D profiles of the selected gel areas.

Determination of Changes in Protein Quantities Based on SYPRO-Ruby-Stained Gels

The fluorescent dye SYPRO Ruby qualifies for quantitative analysis of differences in protein abundance, since it combines a low detection limit with a wide linear range (table 1). The first applications of SYPRO Ruby for 2DE analysis were reported only a few years ago [22, 23]. Since then this protein stain was used for quantitative differential analysis of protein profiles in standard bacteria such as Escherichia coli [5] or environmental bacteria [18]. With respect to detection limit and linear range, SYPRO Ruby is similar to CyDyes. Some differences in the current application protocols and general characteristics of the two types of fluorescent dyes are obvious. (1) Labeling with CyDyes is carried out prior to and staining with SYPRO Ruby after electrophoresis. (2) The availability of different CyDyes and their pre-electrophoretic labeling principle allows to run different samples on a single gel. (3) CyDyes are covalently bound to the $\varepsilon$-amino group of lysine residues, whereas SYPRO Ruby is suggested to interact preferentially with basic amino acids until saturation [24]. (4) Image analysis of CyDye-stained proteins can involve an internal standard, if DeCyder software is used. This is not possible with SYPRO-Rubystained gels. Considering these differences, the question arises whether application of CyDyes and SYPRO Ruby, respectively, has different effects on the changes in protein abundance determined.

We compared these two types of fluorescent dyes in the model system of the present study, namely the inducible $\mathrm{N}$-acetylglucosamine metabolism in Pirellula sp. strain 1. For SYPRO Ruby staining, four gels were analyzed for each of the two studied growth conditions (growth with $\mathrm{N}$-acetylglucosamine or glucose); all 8 gels were run simultaneously. The protein load was $50 \mu \mathrm{g}$ per gel. Image analysis was performed with the ImageMaster software, as described in the Materials and Methods section. Within the set of 24 proteins analyzed the variance of the volumes determined for a single spot in the parallel gels was different. The coefficients of variance for the normalized volumes ranged between $102 \%$ (spot No. 10 in 'Glucose' gels) and $1 \%$ (spot No. 4 in N-acetylglucosamine gels), indicating that the reliability of these values is not comparable. After spot detection, the 24 spots identified by the 2D DIGE process as differently abundant (see preceding paragraph and table 3) were selected for quantitative analysis after normalization. Results (listed in table 3) show that the changes in abundance determined with the two types of fluorescent dyes were overall fairly similar. In most cases, differences in abundance varied 1-1.5-fold. The maximal difference in abundance of 3.7-fold was observed for spot No. 1. These results indicate that the absolute values for changes in abundance of a particular protein can differ, when different types of fluorescent 

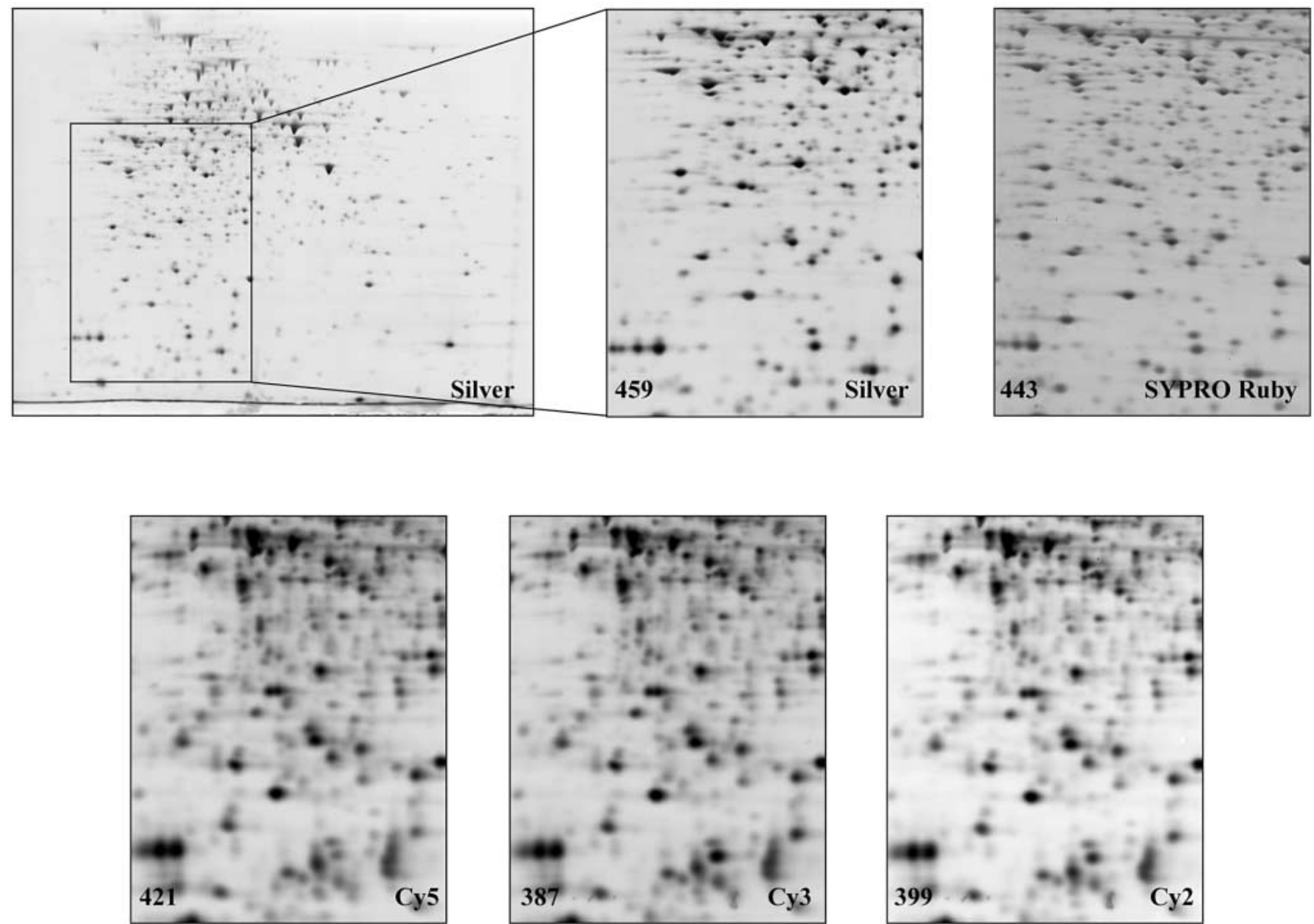

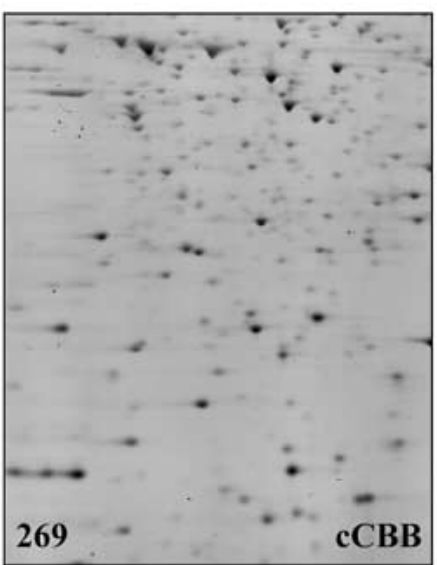

Fig. 4. Partial views of $2 \mathrm{DE}$ gels from protein samples of Pirellula sp. strain 1 stained with different dyes. In each case the total amount of protein used for $2 \mathrm{DE}$ was $50 \mu \mathrm{g}$. Applied stains were silver nitrate, the fluorescent dyes SYPRO Ruby, Cy5, Cy3, Cy2, and cCBB according to Doherty et al. [19], and using the commercial kit Fast Blue. The type of stain is indicated in the bottom right corner of each

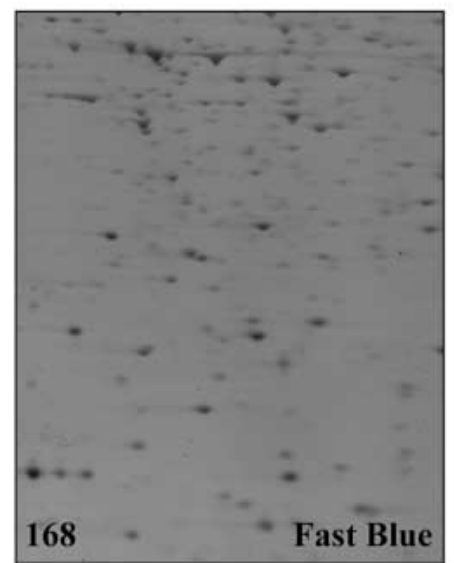

gel section. For each stain, the maximal number of detectable protein spots was determined within the shown section of 3 parallel gels; the average numbers are indicated in the bottom left corner of each gel section. The darker image of the gel section stained with Fast Blue reflects the higher background produced by this staining kit. 
dyes are used. Therefore, within a given experiment, quantitative data derived from different types of stains and/or methods of image analysis should not be mixed.

\section{Sensitivity of CyDyes in Comparison with That of \\ Other Protein Stains}

The sensitivity of protein stains is often defined as the minimum amount of protein detectable with a particular stain (see table 1). Sensitivity can also be understood as the maximal number of protein spots detectable with a particular dye on a $2 \mathrm{DE}$ gel. We evaluated the latter definition of sensitivity for the stains used in this study. For each stain (Cy5, Cy3, Cy2, SYPRO Ruby, silver, and cCBB) we analyzed 3 parallel gel images. To allow optimally comparable conditions, solubilization of proteins was carried out with the lysis buffer specific for labeling with CyDyes and all gels were loaded with $50 \mu$ g protein. After electrophoresis and staining, respectively, the same section in the low molecular range and middle of the $\mathrm{pH}$ gradient (4-7) was selected for spot detection in all gels (fig. 4). In this area of 2DE gels, protein spots are generally separated best and spot boundaries can be defined most easily. Image analysis was performed with the ImageMaster software. For spot detection, the 'spot detection wizard' was used, followed by manual control and adjustments. The maximal number of detectable spots in the selected area was determined for each gel and an average value was calculated from the three parallel gels analyzed for each stain (fig. 4). With 459 and 443 detected spots, silver and SYPRO Ruby appeared to be most sensitive for staining proteins of Pirellula sp. strain 1. The CyDyes allowed detection of only somewhat fewer proteins, namely 418 (Cy5), 399 (Cy2) and 387 (Cy3). This agrees with an earlier report by Tonge et al. [17]. As expected, Coomassie-stained gels revealed lower numbers of detectable spots than the aforementioned stains. However, if gels were stained with cCBB, essentially according to the method described by Doherty et al. [19], considerably more protein spots were detected (269) than with the commercial staining kit (168). As expected from the described detection limits of the used stains (table 1), spots specifically detected with the sensitive dyes were those of low abundance. Comparing these numbers, it should be considered that the sensitivity of a particular stain can vary between different protein species, and that comparison of absolute numbers of detection limits reported in different studies is often hampered by the variety of protocols used for staining.

\section{Conclusions}

The 2D DIGE system together with DeCyder software allows determination of differences in protein abundance. The degree of statistical confidence, however, depends on user-defined parameters, such as the number of parallel gels, or the minimal and maximal values for average ratio and $t$ test, respectively, which are demanded to justify significance. In case of stringent statistical parameters, the number of recognized spots differing in abundance was greatly reduced as compared to the scenario with less stringent parameters. Even if the differences in protein abundance may be significant from an analytical point of view, the biological meaning has to be verified by means of protein identification and correlation with the genomic and physiological context. The comparative analysis of batches of 5 and 10 gels in this study, demonstrated that 5 parallel gels should be sufficient to achieve results with reasonable statistical confidence. To take into account sample-to-sample variations as well, one may also analyze several independent samples obtained under the same biological condition. The co-separation of different samples in combination with an image analysis implementing an internal standard by the 2D DIGE system clearly surpassed the SYPRO Ruby/ImageMaster system with respect to work- and time efficiency as well as statistical confidence.

\section{Acknowledgements}

We would like to thank Rudolf Amann and Friedrich Widdel for general support of proteomic work at our institute. This study was supported by the BMBF, the Max-Planck-Society and Amersham Biosciences. 


\section{References}

1 Klose J. Large-gel 2-D electrophoresis. Methods Mol Biol 112:147-172;1999.

2 Görg A, Obermaier C, Boguth G, Harder A, Scheibe B, Wildgruber R, Weiss W. The current state of two-dimensional electrophoresis with immobilized $\mathrm{pH}$ gradients. Electrophoresis 21:1037-1053;2000.

3 Lauber WM, Carroll JA, Dufield DR, Kiesel JR, Radabaugh MR, Malone JP. Mass spectrometry compatibility of two-dimensional gel protein stains. Electrophoresis 22:906-918; 2001.

4 Patton WF. Detection technologies in proteome analysis. J Chromatogr B 771:3-31; 2002

5 Nishihara JC, Champion KM. Quantitative evaluation of proteins in one- and two-dimensional polyacrylamide gels using a fluorescent stain. Electrophoresis 23:2203-2215;2002.

6 Ünlü M, Morgan ME, Minden JS. Difference gel electrophoresis: A single gel method for detecting changes in protein extracts. Electrophoresis 18:2071-2077;1997.

7 Von Eggeling F, Gawriljuk A, Fiedler W, Ernst G, Claussen U, Klose J, Römer I. Fluorescent dual colour 2D-protein gel electrophoresis for rapid detection of differences in protein pattern with standard image analysis software. Int J Mol Med 8:373-377;2001.

8 Gharbi S, Gaffney P, Yang A, Zvelebil MJ, Cramer R, Waterfield MD, Timms JF. Evaluation of two-dimensional difference gel electrophoresis for proteomic expression analysis of a model breast cancer cell system. Mol Cel Proteomics 1.2:91-98;2002.

9 Skynner HA, Rosahl TW, Knowles MR, Salim K, Reid L, Cothliff R, McAllister G, Guest PC. Alterations of stress related proteins in genetically altered mice revealed by two-dimensional differential in-gel electrophoresis analysis. Proteomics 2:1018-1025;2002.
10 Zhou G, Li H, DeCamps D, Chen S, Shu H, Gong Y, Flaig M, Gillespie JW, Hu N, Taylor PR, Emmert-Buck MR, Liotta LA, Petricoin III EF, Zhao Y. 2D differential in-gel electrophoresis for the identification of esophageal scans cell cancer-specific protein markers. Mol Cell Proteomics 1.2:117-123;2002.

11 Yan JX, Devenish AT, Wait R, Stone T, Lewis $\mathrm{S}$, Fowler S. Fluorescence two-dimensional difference gel electrophoresis and mass spectrometry based proteomic analysis of Escherichia coli. Proteomics 2:1682-1698;2002.

12 Alban A, David SO, Bjorkesten L, Andersson C, Sloge E, Lewis S, Currie I. A novel experimental design for comparative two-dimensional gel analysis: Two-dimensional difference gel electrophoresis incorporating a pooled internal standard. Proteomics 3:36-44;2003.

13 Schlesner H. The development of media suitable for the microorganisms morphologically resembling Planctomyces spp., Pirellula spp., and other Planctomycetales from various aquatic habitats using dilute media. System Appl Microbiol 17:135-145;1994.

14 Rabus R, Gade D, Helbig R, Bauer M, Glöckner FO, Kube M, Schlesner H, Reinhardt R, Amann R. Analysis of $N$-acetylglucosamine metabolism in the marine bacterium Pirellula sp. strain 1 by a proteomic approach. Proteomics 2:649-655;2002.

15 Kirchman DL, White J. Hydrolysis and mineralization of chitin in the Delaware Estuary. Aquat Microb Ecol 18:187-196;1999.

16 Bradford MM. A rapid and sensitive method for the quantitation of microgram quantities of protein utilizing the principle of protein-dye binding. Anal Biochem 72:248-254;1976.

17 Tonge R, Shaw J, Middleton B, Rowlinson R, Rayner S, Young J, Pognan F, Hawkins E, Currie I, Davison M. Validation and development of fluorescence two-dimensional differential gel electrophoresis proteomics technology. Proteomics 1:377-396;2001.

18 Rabus R, Brüchert V, Amann J, Könneke M. Physiological response to temperature changes of the marine, sulfate-reducing bacterium $D e$ sulfobacterium autotrophicum. FEMS Microbiol Ecol 42:409-417;2002.
19 Doherty NS, Littman BH, Reilly K, Swindell AC, Buss JM, Anderson NL. Analysis of changes in acute-phase plasma proteins in an acute inflammatory response and in rheumatoid arthritis using two-dimensional gel electrophoresis. Electrophoresis 19:355-363;1998.

20 Heukeshoven J, Dernick R. Improved silver staining procedure for fast staining in PhastSystem Development Unit. I. Staining of sodium dodecyl sulfate gels. Electrophoresis 9:28$32 ; 1988$.

21 Eason C, Coles CW, Gettinby G. Mathematics and statistics for the biosciences. John Wiley \& Sons, New York; 1989.

22 Berggren K, Chernokalskaya E, Steinberg TH, Kemper C, Lopez MF, Diwu Z, Haugland RP, Patton WF. Background-free, high sensitivity staining of proteins in one- and two-dimensional sodium dodecyl sulfate-polyacrylamide gels using a luminescent ruthenium complex. Electrophoresis 21:2509-2521;2000.

23 Lopez MF, Berggren K, Chernokalskaya E, Lazarev A, Robinson M, Patton WF. A comparison of silver stain and SYPRO Ruby Protein Gel Stain with respect to protein detection in two-dimensional gels and identification by peptide mass profiling. Electrophoresis 21: 3673-3683;2000.

24 Steinberg TH, Chernokalskaya E, Berggren K, Lopez MF, Diwu Z, Haugland RP, Patton WF. Ultrasensitive fluorescence protein detection in isoelectric focusing gels using a ruthenium metal chelate stain. Electrophoresis 21:486496;2000.

25 Yan JX, Wait R, Berkelman T, Harry RA, Westbrook JA, Wheeler CH, Dunn MJ. A modified silver staining protocol for visualization of proteins compatible with matrix-assisted laser desorption/ionization and electrospray ionization-mass spectrometry. Electrophoresis 21: 3666-3672;2000. 